\title{
Mal de Pott y diagnóstico inmunológico de tuberculosis, a propósito de un caso pediátrico
}

\author{
Verónica Contardo, José Cofré y Pamela Hernández
}

\section{Pott's disease and immunological diagnosis of tuberculosis, in relationship to a pediatric clinical case}

Tuberculosis (TB) remains a major health problem in the world. The clinical forms of TB in children are variable, pulmonary involvement occurs in two thirds of cases. In the remaining third, clinical forms incluye node, meningeal and osteoarticular involvement. Case report: 7 year old boy with a history of an osteolytic lesion of the right ischial branch. Three months later he presented with spondylodiscitis at L2-L3, associated with a large abscess in the right iliac psoas muscle. Pott's disease was suspected, and tuberculin test and T-SPOT ${ }^{\circledR}$.TB test were performed, with a positive result. Antimicrobial treatment was initiated with isoniazid, rifampicin, pyrazinamide and ethambutol. After 30 days, Mycobacterium tuberculosis was isolated from psoas abscess. We discuss methods of TB diagnosis, with special emphasis on immunological methods: tuberculin test and interferon-gamma release assays. Methods of immunological TB diagnosis are an important contribution to the diagnosis of this disease, allowing early initiation of treatment.

Key words: Tuberculosis, Pott's disease, tuberculin test, interferon-gamma release assays.

Palabras clave: Tuberculosis, mal de Pott, reacción de tuberculina, test de liberación de interferón-gamma.

\section{Introducción}

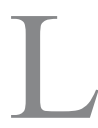

a tuberculosis (TBC) continúa siendo un importante problema de salud en el mundo. En el año 2012 se estima que 8,6 millones de personas desarrollaron TBC y 1,3 millones fallecieron por esta enfermedad $^{1}$. En Chile, la incidencia de TBC ha disminuido durante las últimas tres décadas; sin embargo, en los últimos siete años no ha presentado mayor variación, manteniéndose entre 12 y 13 por 100 mil habitantes ${ }^{2}$. Además, presenta una importante variabilidad territorial, destacando los Servicios de Salud de Iquique y Arica con incidencias máximas de 32,2 y 31,4 por 100 mil habitantes, respectivamente, y los servicios de Maule y Aconcagua con incidencias mínimas de 8,1 y 6,0 por 100 mil, respectivamente, durante el año 2012. Los casos se concentran en la población entre 15 y 64 años, con 75,7\% del total. La población infantil representa sólo $2,2 \%$ de los $\operatorname{casos}^{2}$.

Las formas clínicas de TBC en los niños son muy variadas, presentándose compromiso pulmonar en dos tercios de los casos. En el tercio restante destacan los compromisos ganglionar, meníngeo y osteoarticular ${ }^{2}$.

A fines del siglo XVIII, Percival Pott describió el mal de Pott, caracterizado por paraplejia, caries vertebrales, giba y abscesos fríos causados por Mycobacterium tuberculosis. Éste se produce cuando la infección, que se desarrolla a partir de la sinovial articular de la columna vertebral, invade y destruye el disco y el hueso esponjoso, produciendo el colapso vertebral. Las colecciones paravertebrales pueden desarrollarse a nivel cervical, dorsal o lumbar, según cuál sea la vértebra comprometida. En las vértebras lumbares, los abscesos siguen la vaina del psoas, deslizándose entre el plano muscular y retroperitoneo, prolongándose hacia la fosa ilíaca y formando colecciones en la inserción baja del psoas (triángulo femoral y trocánter menor).

Describimos el caso clínico de un niño que presentó un mal de Pott asociado a un absceso del músculo psoasilíaco derecho y discutimos los métodos de diagnóstico inmunológico de TBC en pediatría que permitieron establecer con mayor certeza la etiología del cuadro.

\section{Caso Clínico}

Varón de 7 años de edad, previamente sano, proveniente de Alto Hospicio (Región de Tarapacá) con antecedentes de madre boliviana que residía en Chile hacía más de 10 años. Período perinatal normal y calendario nacional de vacunas al día. Sin antecedente de viajes al exterior ni contacto con pacientes tuberculosos conocidos, tosedores crónicos, o gatos. Su dolencia se remontaba a un año padeciendo de dolor lumbar, coxalgia derecha y claudicación de la marcha. Durante este período se le realizó una radiografía de columna vertebral que mostró
Universidad de Chile, Facultad de Medicina, Programa de Sub-especialidad en Infectología Pediátrica (VC). Hospital Luis Calvo Mackenna, Servicio de Pediatría (PH). Unidad de Infectología (JC).

Los autores declaran no tener conflictos de interés ni fuente de financiamiento.

Recibido: 25 de agosto de 2014 Aceptado: 19 de enero de 2015

Correspondencia a: Verónica Contardo Pérez vcontardo@gmail.com 

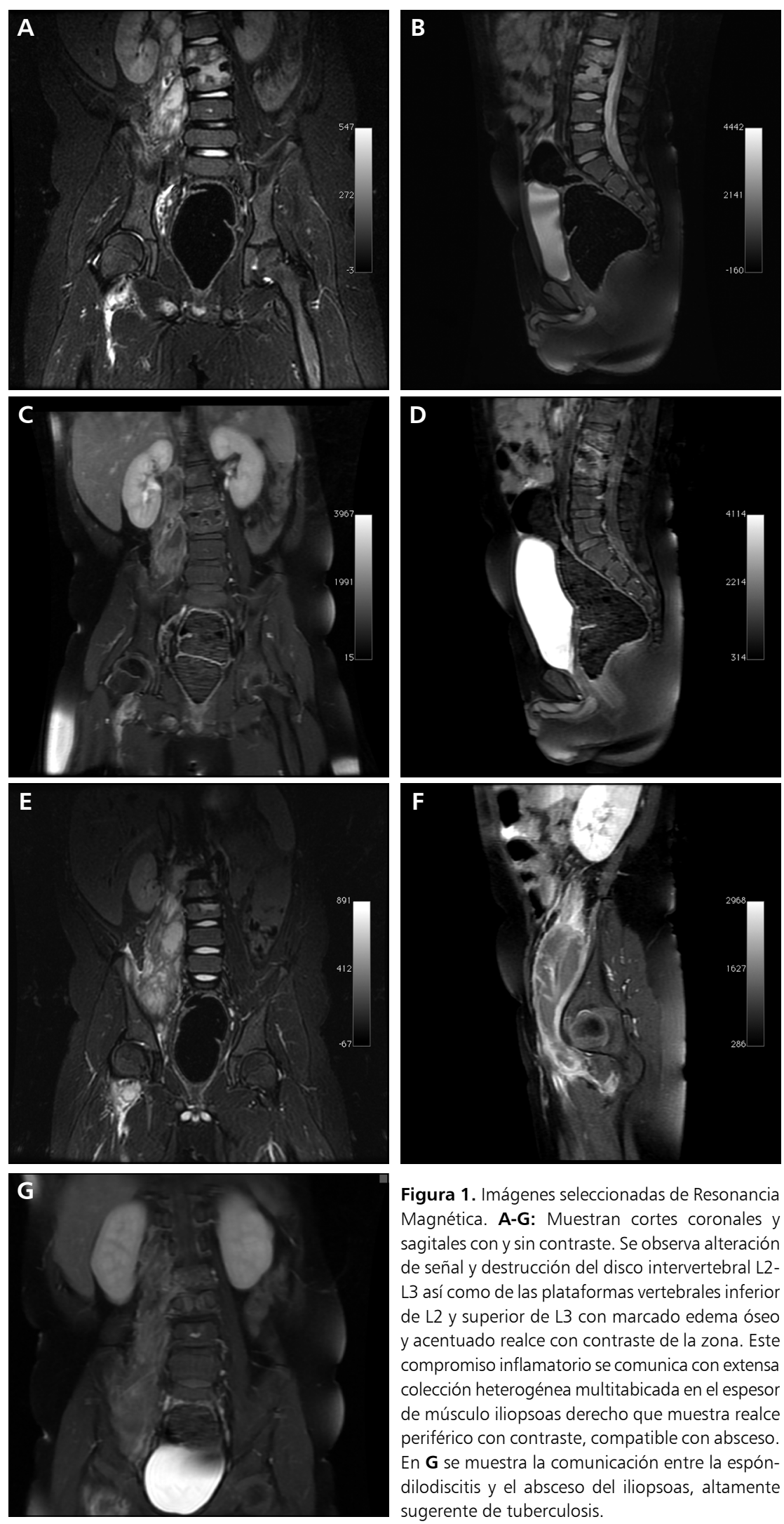
de L2 y superior de L3 con marcado edema óseo y acentuado realce con contraste de la zona. Este compromiso inflamatorio se comunica con extensa colección heterogénea multitabicada en el espesor de músculo iliopsoas derecho que muestra realce periférico con contraste, compatible con absceso. En $\mathbf{G}$ se muestra la comunicación entre la espóndilodiscitis y el absceso del iliopsoas, altamente sugerente de tuberculosis.

una xifo-escoliosis dorsolumbar asociada a una lesión expansiva de la rama isquio-pubiana derecha. El estudio se complementó con una RM pélvica que mostró una lesión osteolítica de la rama isquiática derecha, de 5,6 cm de diámetro, con compromiso de los tejidos blandos adyacentes. Con la hipótesis de un tumor, se le realizó una biopsia cuya histología fue compatible con una osteomielitis crónica granulomatosa. Recibió tratamiento con flucloxacilina durante seis semanas; sin embargo, los síntomas persistieron. Tres meses después, una nueva radiografía evidenciaba una imagen lítica inespecífica en L2-L3. Al repetir la RM, se observó una espondilodiscitis en L2-L3, asociada a un gran absceso en el músculo psoas-ilíaco derecho, por lo que se decidió su internación (Figura 1).

Al examen físico se encontraba en buen estado general, afebril, pálido, la piel sin lesiones, ausencia de cicatriz BCG y sin adenopatías palpables. Presentaba una escoliosis leve, dolor a la palpación de la columna dorso-lumbar y una leve claudicación al caminar. Se diagnosticó una espondilodiscitis L2-L3, con sospecha de mal de Pott asociado a un absceso del psoas-ilíaco derecho. Entre los exámenes de laboratorio destacaban: recuento de leucocitos de 9.500 céls $/ \mathrm{mm}^{3}$ (65\% segmentados y $20 \%$ linfocitos), hematocrito $31 \%$, hemoglobina $10 \mathrm{~g} / \mathrm{dL}$, recuento de plaquetas de $353.000 / \mathrm{mm}^{3}$, PCR $45 \mathrm{mg} / \mathrm{L}$ y VHS $69 \mathrm{~mm} / \mathrm{h}$. Radiografía de tórax normal y TAC torácica con adenopatías calcificadas perihiliares bilaterales e infracarinales, sugerentes de una TBC antigua (Figura 2). Se efectuó serología Ig G para Bartonella henselae y ELISA VIH que resultaron negativos. Se procedió a puncionar el absceso del psoas guiado por una ecotomografía, drenándose $80 \mathrm{ml}$ de secreción purulenta. De esta muestra se realizó tinción de Gram directo, cultivo corriente, tinción con calcoflúor, cultivo de hongos, baciloscopia y RPC para complejo M. tuberculosis, todos con resultado negativo. Las baciloscopias de aspirado gástrico fueron negativas. Tanto el PPD -de 2 UT- $(20 \mathrm{~mm})$ como la determinación de T-SPOT ${ }^{\circledR}$.TB, resultaron positivos. Se diagnosticó un mal de Pott y se inició terapia asociada con isoniazida, rifampicina, pirazinamida y etambutol. Además se le estabilizó la columna con corset. Después de 30 días, en el Instituto Nacional del Tórax, se aisló $M$. tuberculosis del absceso del psoas, el que posteriormente se confirmó en el Instituto de Salud Pública, sin realizarse pruebas de susceptibilidad.

El paciente completó su tratamiento antituberculoso y evolucionó satisfactoriamente.

\section{Discusión}

La infección por M. tuberculosis es una enfermedad de difícil diagnóstico en pediatría. Esto se debe a las dificultades que existen para realizar un aislamiento 

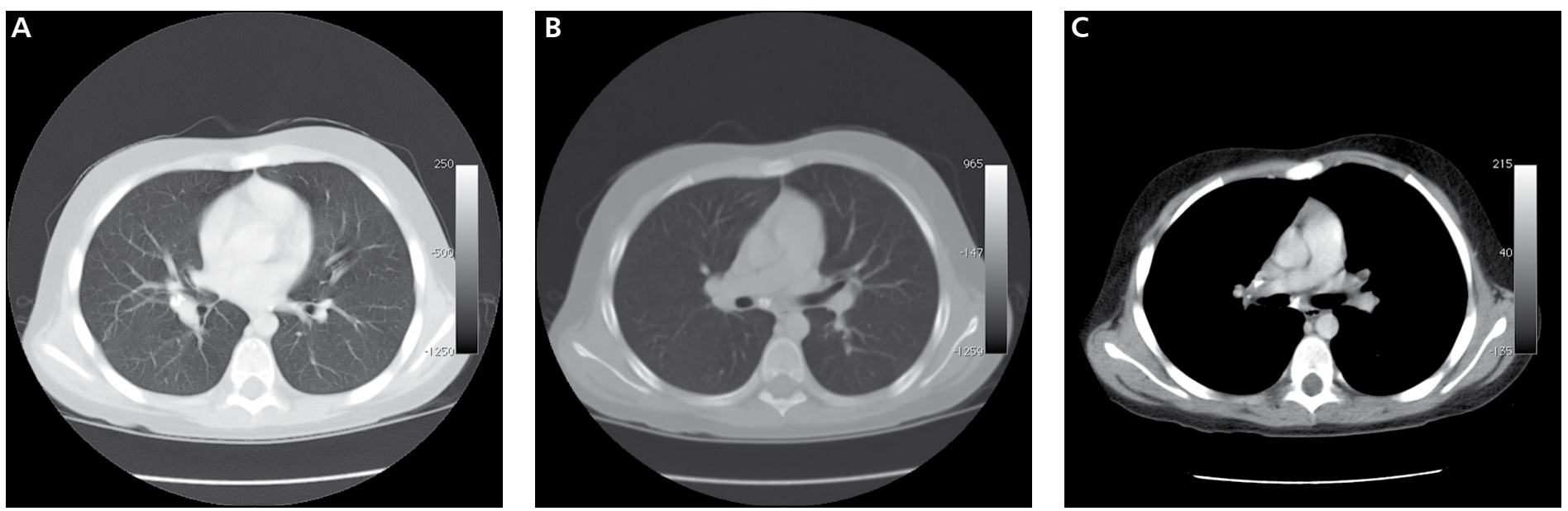

Figura 2. Imágenes seleccionadas de cortes axiales de tomografía computarizada de tórax. A, B: En ventana pulmonar se observan adenopatías calcificadas en ubicación subcarinal a izquierda e hiliares bilaterales. C: En ventana mediastínica se observan adenopatías calcificadas en el mediastino (paratraqueal derecha y subcarinal).

microbiológico oportuno, debido al lento crecimiento que presenta M. tuberculosis. Los cultivos en medio líquido han disminuido el tiempo de espera a la mitad, manteniendo similar sensibilidad; sin embargo, no siempre es posible obtener una muestra óptima. Debido a que las infecciones en pediatría generalmente no son bacilíferas, las baciloscopias de expectoración y contenido gástrico suelen no ser útiles. El estudio molecular de la TBC ha significado un gran avance; destaca Xpert ${ }^{\circledR}$ MTB/ RIF, que además de detectar material genético de $M$. tuberculosis de forma completamente automatizada, es capaz de identificar resistencia a rifampicina. Un reciente metanálisis realizado por el grupo Cochrane encontró que su sensibilidad para TBC pulmonar en adultos fue de $89 \%$ y su especificidad de $99 \%$. Sin embargo, cuando la baciloscopia es negativa, su sensibilidad disminuye a $67 \%{ }^{3}$. Para el diagnóstico de TBC pulmonar en niños, la mejor sensibilidad fue de $71 \%$ cuando se realizaba Xpert® MTB/RIF de dos muestras de esputo inducido y $65 \%$ cuando las muestras eran de hisopado nasofarínge $\mathrm{o}^{4}$. Para TBC extrapulmonar la sensibilidad de este mismo método fue analizada recientemente. Se encontró que para adenopatía, líquido cefalorraquídeo y líquido pleural era de $81,2 \%, 80,5 \%$ y $46,4 \%$; respectivamente al compararlo con cultivo, manteniendo en todos estos una especificidad mayor a $98 \% \%^{5}$.

El estudio histológico es otro elemento útil para orientar al diagnóstico de TBC. Este se caracteriza por una reacción inflamatoria crónica, con formación de granulomas, que si bien no es específico, obliga a descartar esta etiología ${ }^{6}$. Lamentablemente en nuestro paciente este diagnóstico no fue considerado en la primera evaluación por traumatología.

Las pruebas inmunológicas siguen teniendo un rol protagónico en el diagnóstico de la tuberculosis. La reacción de tuberculina o PPD (purified proteic derivative) evalúa la hipersensibilidad tardía frente a la inyección intradérmica de antígenos de la pared celular de $M$. tuberculosis ( 2 unidades de tuberculina) ${ }^{7}$. Se considera positivo este test en el individuo inmunocompetente si, tras 48-72 h de efectuada la inoculación, se observa un diámetro de induración $\geq 10 \mathrm{~mm}$. En el caso de los pacientes inmunocomprometidos -como infectados por VIH o usuarios de inmunomoduladores- se considera positivo si esta induración es $>5 \mathrm{~mm}$. Las ventajas de este método son su bajo costo y su disponibilidad en nuestros hospitales; sin embargo, presenta debilidades tales como: requiere de un profesional entrenado específicamente en su ejecución y su lectura; esta lectura debe efectuarse, en forma estandarizada, a las 48-72 h de inoculado el antígeno; en caso de una primoinfección, se torna positivo recién entre las tres a cuatro semanas de exposición; la sensibilización a $M$. tuberculosis puede durar toda la vida, indicando sólo sensibilización a antígenos y no necesariamente enfermedad. Una desventaja destacable es su baja especificidad en pacientes con vacuna BCG. Sarah Burl y cols. ${ }^{8}$, describieron que $27 \%$ de los lactantes sanos de 4 meses y medio presentaban un PPD $>10 \mathrm{~mm}$ y al ser re-evaluados a los 20 a 28 meses de vida, éste se mantenía reactivo en $5 \%$. En otro estudio, Bozaykut y cols. ${ }^{9}$, comunicaron que $23 \%$ de los niños sanos de 2 años de edad presentaban un PPD de $10 \mathrm{~mm}$, lo que se redujo a $15 \%$ cuando se evaluaron niños de 6 años de edad. Chan y cols. ${ }^{10}$, evaluaron el PPD en niños sanos de diferentes grupos etarios, encontrando un PPD $>10 \mathrm{~mm}$ en $40 \%$ de 166 niños entre 3 meses y 3 años de edad, $8,5 \%$ en 156 niños de 4 a 7 años y en $27 \%$ de 461 niños entre 8 y 14 años de edad. Estos datos muestran que un 
PPD reactivo es habitual en niños sanos vacunados con BCG, como sucede en nuestro país. Sin embargo, existe una amplia variedad de resultados de PPD en relación a las múltiples vacunas BCG disponibles ${ }^{11}$.

En un meta-análisis, Pai y cols. ${ }^{12}$, describieron que la especificidad del PPD difería en forma importante en pacientes vacunados comparados con personas no vacunadas, siendo de 59\% (46 a 73\%) en individuos con BCG y de $97 \%$ en quienes no habían recibido esta vacuna. Este mismo meta-análisis encontró una sensibilidad de $77 \%$ (71 a $82 \%$ ) para el PPD.

La especificidad del PPD también puede ser baja, debido a la presencia de infecciones producidas por micobacterias no tuberculosas (MNT). En un estudio realizado Alemania, en población pediátrica $(95 \%$ no vacunada con BCG), la especificidad del PPD fue de $58 \%$, dado que también se encontraba reactivo $>10 \mathrm{~mm}$ en $78 \%$ de los niños infectados por $\mathrm{MNT}^{13}$. En Chile, según el Instituto de Salud Pública, 8,4\% de las micobacterias aisladas de muestras pulmonares y extrapulmonares corresponden a micobacterias atípicas ${ }^{14}$.

Se sabe que la sensibilidad de la intradermo-reacción disminuye en algunas circunstancias como inmunodeficiencias celulares (linfoma, leucemia, VIH sin tratamiento), exposición a vacunas que contienen virus vivos atenuados, corticoterapias prolongadas, durante algunas infecciones virales, TBC avanzada, desnutrición, o edades extremas. También puede ser menor en infecciones extrapulmonares, como lo describe Wu y cols. ${ }^{15}$, al encontrar que sólo $61 \%$ de los niños con infección extra-pulmonar (meníngea, diseminada, abdominal, osteo-articular o linfática) presentaron un PPD reactivo en comparación a $80 \%$ en las TBC pulmonares.

Otra característica descrita del PPD es el efecto booster, lo que puede ser una ventaja en el estudio de TBC latente en inmunocomprometidos o pacientes con infección por VIH, pero es una desventaja para estudiar a pacientes pediátricos vacunados con $\mathrm{BCG}$, porque podría estimular la respuesta inmune al PPD en una segunda medición, disminuyendo más aún su especificidad.

Con la idea de superar las debilidades del PPD mencionadas anteriormente, surgieron los test de liberación de interferón gamma o IGRAs (del inglés Interferon Gamma Release Assays), inmunoensayos que miden la liberación de interferón gamma (IF- $\gamma$ ) producidos por linfocitos $\mathrm{T}$ sensibilizados por los antígenos de M. tuberculosis. El primero en desarrollarse y ser aprobado por la FDA (2001) fue QuantiFERON TB ${ }^{\circledR}$, que medía por el método de ELISA la cantidad de IF- $\gamma$ en respuesta a derivado proteico purificado de la pared celular de M. tuberculosis comparado con controles. Sin embargo, la especificidad de este método fue menor que el PPD, razón por la cual fue retirado cuatro años después del mercado ${ }^{16}$. Para superar esta debilidad se desarrolló una nueva generación de IGRAs, que evalúan la respuesta a péptidos sintéticos que representan proteínas específicas de $M$. tuberculosis y estimulan la liberación medible de IF- $\gamma$ en personas infectadas, pero están ausentes en la cepa vaccinal BCG y en la mayoría de las MNT.

Los métodos actualmente disponibles en nuestro medio son QuantiFERON TB Gold ${ }^{\circledR}$ In Tube (QFT-GIT), que evalúa la respuesta a los antígenos ESAT-6, CFP-10 y TB7.7 (considerándose positivo un valor $\geq 0,35 \mathrm{UI} /$ $\mathrm{ml}$ y mayor a $25 \%$ del control negativo) y el T-SPOT ${ }^{\circledR}$. TB que evalúa la respuesta a ESAT-6 y CFP-10. Este último no mide la concentración de IF- $\gamma$ liberado, sino que el número de células que lo liberan en respuesta a estos antígenos, siendo positivo un valor de ocho o más manchas (del inglés spots) y límite entre cinco y siete manchas $^{16}$.

Las principales ventajas de los IGRAs son: que no se afectan por BCG ni micobacterias ambientales, a excepción de $M$. kansasii, M. szulgai y M. marinum que corresponden solo a $2,4 \%$ de las micobacterias aisladas en Chile ${ }^{14}$, implican sólo una visita, no generan reacciones adversas ni requieren ser interpretadas por un examinador. Sus principales desventajas son su alto costo, su menor disponibilidad actualmente en los hospitales públicos, que no diferencian infección activa/latente versus enfermedad y finalmente que los datos son limitados en niños bajo 5 años de edad. Diferentes meta-análisis efectuados en adultos sin infección por VIH, encuentran que el QTFGIT tiene una sensibilidad alrededor de $80 \%$ (74-84\%) y el T-SPOT ${ }^{\circledR}$.TB alrededor de $90 \%(87,5-91 \%)^{16-18}$. Dos meta-análisis que incluyeron sólo estudios en niños, encontraron una sensibilidad de $83-79 \%$ para QTF-GIT y $84-74 \%$ para T-SPOT $^{\circledR}$.TB, respectivamente ${ }^{19,20}$. Esta disminución de la sensibilidad podría deberse a un aumento de resultados indeterminados en los niños bajo 5 años de edad, probablemente secundario a la inmadurez inmunológica que presentan a esta edad, planteándose que los valores de corte no son necesariamente correctos para su uso en pediatría ${ }^{21,22}$. La especificidad encontrada en niños para QTF-GIT es de $95 \%$ y para T-SPOT ${ }^{\circledR}$.TB de $96 \%{ }^{20}$. Un meta-análisis publicado recientemente, diferencia los resultados encontrados en países de altos y bajos ingresos, destacando en estos últimos, la menor sensibilidad de ambos test (57\% para QTF-GIT y $61 \%$ para T-SPOT ${ }^{\circledR}$.TB $)^{23}$.

Herrmann y cols., estudiaron la respuesta temporal del QTF-GIT en niños y encontraron que en TBC activa se mantiene positivo hasta el día 180 de evolución, a pesar de recibir un tratamiento adecuado, presentando su mayor alza al día $10^{24}$.

En relación al uso de los IGRAs, la Academia Americana de Pediatría recomienda que:

- El PPD es el test preferido, pero IGRAs son aceptables, en niños bajo 5 años de edad. 
- Los tests IGRAs son preferidos, pero PPD es aceptable en niños de 5 años o más con BCG y/o que probablemente no regresen para la lectura del PPD.

- Realizar ambos métodos (PPD más IGRA) deben ser considerados cuando: el IGRA inicial y repetido es indeterminado, el test inicial (IGRA o PPD) resulta negativo y hay alta sospecha clínica de TBC o un alto riesgo de progresión y pobre pronóstico, o cuando el PPD es positivo en un niño mayor de 5 años con BCG. Además se recomienda si hay necesidad de evidencia adicional para aumentar la adherencia o en casos de sospecha de enfermedad por MNT ${ }^{16,25}$.

Las guías europeas mencionan que los IGRAs serían un aporte en el diagnóstico de TBC extra-pulmonar, pacientes con baciloscopia y cultivo negativos, diagnóstico diferencial con MNT, pacientes con infección por VIH y diagnóstico de $\mathrm{TBC}$ en niños ${ }^{26}$.

Otros elementos a considerar al momento de recomendar su uso son las características de cada país, siendo recomendado su uso en los países de altos ingresos y baja incidencia. De lo contrario, el primer método de diagnóstico recomendado sería el $\mathrm{PPD}^{27}$. Sin embargo, existe una gran diversidad de recomendaciones en la literatura médica.

Los IGRAs se encuentran actualmente disponibles en nuestro medio y están siendo utilizados tanto en la práctica clínica, como en investigación ${ }^{28}$.

Actualmente se encuentran en estudio los IGRAs de cuarta generación, que incorporan la medición de IF- $\gamma$, secretado frente a antígenos adicionales o biomarcadores adicionales, como IP-10, IL-2 y MCP-2.

También se encuentra en investigación para el diagnóstico de TBC activa el análisis de la expresión de ARN de células sanguíneas del hospedero a través de microarray, con resultados preliminares promisorios ${ }^{29}$.

Volviendo a nuestro caso clínico, se consideró necesario realizar un T-SPOT ${ }^{\circledR}$.TB dado que se trataba de un niño sobre 5 años de edad, con un cuadro clínico muy sugerente de infección tuberculosa, un PPD reactivo, pero con el antecedente de haber recibido la vacuna $\mathrm{BCG}$ al nacer (a pesar de no tener cicatriz BCG, respuesta observada en hasta $10 \%$ de los vacunados) y una RPC negativa para
Complejo M. tuberculosis; se buscó así otro elemento más para decidir el inicio del tratamiento anti-tuberculoso.

Podemos concluir que los métodos de diagnóstico inmunológico son una herramienta de gran utilidad para el diagnóstico de TBC, principalmente cuando nos encontramos en un país con vacunación global con BCG y baja incidencia de TBC. La elección del método dependerá de cada paciente. Nosotros recomendamos realizar inicialmente un PPD. Si el resultado es positivo y el niño recibió vacuna BCG, lo ideal sería confirmarlo con un QTF-GIT o un T-SPOT ${ }^{\circledR}$.TB, debido a su alta especificidad. Si el resultado del PPD es negativo y existe una alta sospecha clínica de TBC, principalmente extra-pulmonar, el niño es de alto riesgo de progresión, es inmunocomprometido o tiene una infección por VIH, recomendamos también complementar el estudio con un IGRA, mejorando así la certeza diagnóstica en los pacientes (destacando si que se debe ser cauto en la interpretación de los exámenes en estos dos últimos grupos). Esto permitirá el pronto inicio de tratamiento específico.

Agradecimientos: a Dr. Javier Willatt H.

\section{Resumen}

La tuberculosis sigue siendo un importante problema en salud en el mundo. Las formas clínicas de TBC en los niños son muy variadas, presentándose en dos tercios de los casos compromiso pulmonar. En el tercio restante destacan los compromisos ganglionar, meníngeo y osteoarticular. Caso clínico: Varón de 7 años que presentó una espondilodiscitis L2-L3, asociada a un absceso en músculo psoas-ilíaco derecho. Por sospecha de mal de Pott se realizó PPD y T-SPOT ${ }^{\circledR}$.TB que resultaron positivos. Se inició tratamiento antimicrobiano asociado con isoniazida, rifampicina, pirazinamida y etambutol. Después de 30 días, se aisló Mycobacterium tuberculosis del absceso del psoas. Se discute los métodos de diagnóstico de TBC en pediatría, con especial énfasis en los métodos inmunológicos: reacción de tuberculina y test de liberación de interferón-gamma, los que son una importante contribución para el diagnóstico de esta enfermedad, permitiendo el pronto inicio de su tratamiento.

\section{Referencias bibliográficas}

1.- World Health Organization. Global tuberculosis report 2013. Disponible en: http://apps.who.int/ iris/bitstream/10665/91355/1/9789241564656 eng.pdf?ua $=1$ (accedido: 1 de Septiembre de 2014).

2.- Tuberculosis Informe de Situación Chile: 2012. Ministerio de Salud, Gobierno de Chile. Disponible en: http://web.minsal.cl/sites/
default/files/files/tuberculosisInforme2012.pdf (accedido: 1 de Septiembre de 2014).

3.- Steingart K R, Schiller I, Horne D J, Pai M, Boehme C C, Dendukuri N. Xpert® MTB/ RIF assay for pulmonary tuberculosis and rifampicin resistance in adults. Cochrane Database Syst Rev 2014; 1: CD009593. doi: 10.1002/14651858.CD009593.pub3.

4.- Zar H J, Workman L, Isaacs W, Munro J, Black F, Eley B, et al. Rapid molecular diagnosis of pulmonary tuberculosis in children using nasopharyngeal specimens. Clin Infect Dis 2012; 55: 1088-95.

5.- Denkinger C M, Schumacher S G, Boehme C C, Dendukuri N, Pai M, Steingart K. Xpert MTB/ RIF assay for the diagnosis of extrapulmonary tuberculosis: a systematic review and metaanalysis. Eur Respir J 2014; 44: 435-46.

6.- Starke J. Mycobacterium tuberculosis. In: Long S, Pickering L, Prober C. Principles and Practice 
of Pediatric Infectious Diseases, fourth edition. Elsevier Saunders. 2012, pág 771-86.

7.- Programa nacional de control de tuberculosis. Manual de Organización y Normas Técnicas 2005. Ministerio de Salud, Gobierno de Chile. Disponible en: http://web.minsal.cl/portal/url/it em/803048171acc60f8e04001011f0148e2.pdf (accedido: 1 de Septiembre de 2014).

8.- Burl S, Adetifa U J, Cox M, Touray E, Whittle $\mathrm{H}, \mathrm{McShane} \mathrm{H}$, et al. The tuberculin skin test (TST) is affected by recent BCG vaccination but not by exposure to nontuberculosis mycobacteria (NTM) during early life. PLoS One 2010; 5: e12287.

9.- Bozaykut A, Ipek I O, Ozkars M Y, Seren L P, Atay E, Atay Z. Effect of BCG vaccine on tuberculin skin tests in 1-6-year-old children. Acta Paediatr 2002; 91: 235- 8.

10. Chan P C, Chang L Y, Wu Y C, Lu C Y, Kuo H $\mathrm{S}$, Lee C Y, et al. Age-specific cut-offs for the tuberculin skin test to detect latent tuberculosis in BCG-vaccinated children. Int J Tuberc Lung Dis 2008; 12: 1401-6.

11.- Menzies D. What does tuberculin reactivity after bacille Calmette-Guérin vaccination tell us?. Clin Infect Dis 2000; 31(Suppl 3): S71-4.

12.- Pai M, Zwerling A, Menzies D. T-cell-based assays for the diagnosis of latent tuberculosis infection: an update. Ann Intern Med 2008; 149: 177-84.

13.- Detjen A K, Keil T, Roll S, Hauer B, Mauch H, Wahn U, et al. Interferon-gamma release assays improve the diagnosis of tuberculosis and nontuberculous mycobacterial disease in children in a country with a low incidence of tuberculosis. Clin Infect Dis 2007; 45: 322-8.

14.- Scappaticcio A, Velasco R, Leiva T, Rodríguez J. Frecuencia de micobacterias ambientales en Chile en el año 2008. Rev Chil Enf Respir 2011; 27: 214-22.
15.- Wu X R, Yin Q Q, Jiao A X, Xu B P, Sun L, Jiao W W, et al. Pediatric tuberculosis at Beijing Children's Hospital: 2002-2010. Pediatrics 2012; 130: e1433-40.

16.- Centers for Disease Control and Prevention. Updated guidelines for using interferon gamma release assays to detect Mycobacterium tuberculosis infection. MMWR 2010; 59 (RR-5): 1-13. Disponible en: http://www.cdc. gov/mmwr/pdf/rr/rr5905.pdf (accedido: 1 de septiembre de 2014).

17.- Diel R, Loddenkemper R, Nienhaus A. Evidence-based comparison of commercial interferon-gamma release assays for detecting active TB: a meta-analysis. Chest 2010; 137: 952-68.

18.- Metcalfe J Z, Everett C K, Steingart K R, Cattamanchi A, Huang L, Hopewell P C, et al. Interferon- $\gamma$ release assays for active pulmonary tuberculosis diagnosis in adults in low-and middle-income countries: systematic review and meta-analysis. J Infect Dis 2011; 204 (Suppl 4): S1120-9.

19.- Mandalakas A M, Detjen A K, Hesseling A C, Benedetti A, Menzies D. Interferon-gamma release assays and childhood tuberculosis: systematic review and meta-analysis. Int $\mathrm{J}$ Tuberc Lung Dis 2011; 15: 1018-32.

20.- Chiappini E, Accetta G, Bonsignori F, Boddi V, Galli L, Biggeri A, et al. Interferon- $\gamma$ release assays for the diagnosis of Mycobacterium tuberculosis infection in children: a systematic review and meta-analysis. Int J Immunopathol Pharmacol 2012; 25 : 557-64.

21.- Ferrara G, Losi M, D’Amico R, Roversi P, Piro R, Meacci M, et al. Use in routine clinical practice of two commercial blood tests for diagnosis of infection with Mycobacterium tuberculosis: a prospective study. Lancet 2006; 367: 1328-34.
22.- Kampmann B, Tena-Coki G, Anderson S. Blood tests for diagnosis of tuberculosis. Lancet 2006; 368: 282.

23.- Sollai S, Galli L, De Martino M, Chiappini E. Systematic review and meta-analysis on the utility of Interferon-gamma release assays for the diagnosis of Mycobacterium tuberculosis infection in children: a 2013 update. BMC Infect Dis 2014; 14 (Suppl 1): S6

24.- Herrmann J L, Belloy M, Porcher R, Simonney $\mathrm{N}$, Aboutaam R, Lebourgeois M, et al. Temporal dynamics of interferon gamma responses in children evaluated for tuberculosis. PLoS One 2009; 4: e4130.

25.- American Academy of Pediatrics. Tuberculosis. In: Pickering LK, Baker CJ, Kimberlin DW, Long SS, eds. Red Book: 2012 Report of the Committee on Infectious Diseases. Elk Grove Village, IL; American Academy of Pediatrics; 2012: 736-59.

26.- European Centre for Disease Prevention and Control. Use of interferon-gamma release assays in support of TB diagnosis. Stockholm: ECDC 2011. Disponible en: http://ecdc.europa.eu/en/ publications/publications/1103_gui_igra.pdf (accedido: 1 de septiembre de 2014).

27.- Denkinger C M, Dheda K, Pai M. Guidelines on interferon-g release assays for tuberculosis infection: concordance, discordance or confusion?. Clin Microbiol Infect 2011; 17: 806-14.

28.- Hernández M, Casar C, García P, Morales V, Mamani N, Gómez-Cofré N, et al. Pesquisa de infección tuberculosa latente en personal de la salud en cuatro instituciones de Santiago de Chile. Rev Chilena Inf 2014; 31: 254-60.

29.- Anderson S T, Kaforou M, Brent A J, Wright V J, Banwell C M, Chagaluka G, et al. Diagnosis of childhood tuberculosis and host RNA expression in Africa. N Engl J Med 2014; 370:1712-23. 